\title{
Les réacteurs nucléaires du futur : quatrième génération et SMR
}

\section{Editorial}

F. GAUCHÉ, CEA/DEN, Chef du programme "Réacteurs de quatrième génération"

J. GUIDEZ, CEA/DEN, Président du groupe ST7 en 2014

F. TERNON-MORIN, EDF, Présidente du groupe ST7 en 2013

Les journées ST7 de décembre étaient consacrées aux réacteurs du futur, et comportaient trois volets : les réacteurs de troisième génération, les réacteurs de quatrième génération et les petits réacteurs modulaires (les SMR).

Le RGN N¹ de 2014 a présenté le panorama des réacteurs de troisième génération qui seront l'essentiel de la flotte nucléaire de ce siècle. Ce RGN fait un point sur les réacteurs de quatrième génération en construction ou en développement dans le monde, ainsi que sur les principaux projets de petits réacteurs modulaires (SMR).

\section{Pourquoi des systèmes de $4^{\text {ème }}$ génération ?}

Les réacteurs première génération ont été des réacteurs prototypes permettant de mettre au point les réacteurs actuellement en fonctionnement qui sont des réacteurs dits de seconde génération. Ce sont essentiellement des réacteurs refroidis par de l'eau : bouillants ou pressurisés. Le retour d'expérience et l'évolution des critères de sûreté, ont conduit à la mise au point de réacteurs évolutionnaires qui sont les réacteurs à eau de troisième génération dont
l'EPR est un bon exemple. Les réacteurs de quatrième génération sont des réacteurs en rupture de concept, permettant d'apporter un certain nombre d'avantages, et en particulier pour la plupart d'entre eux, grâce à un spectre de neutrons rapides.

L'article "Les réacteurs rapides, clés d'une énergie nucléaire durable" explique l'intérêt de ces réacteurs rapides pour assurer la durabilité du nucléaire sur des millénaires tout en minimisant les déchets produits : possibilité d'utiliser environ 100 fois mieux la ressource en uranium, possibilité de recycler le plutonium sans limitation du nombre de cycles, éventuelle transmutation de certains actinides mineurs.

Les autres objectifs fixés pour les systèmes de 4ème génération sont :

- avoir un niveau de sûreté à minima équivalent aux

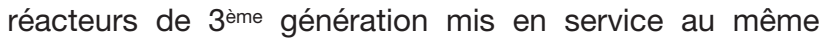
moment. Pour le démonstrateur ASTRID, cela signifie un niveau de sûreté au moins équivalent aux réacteurs de 3 ème génération, auquel seront intégrés les enseignements de l'accident de Fukushima ;

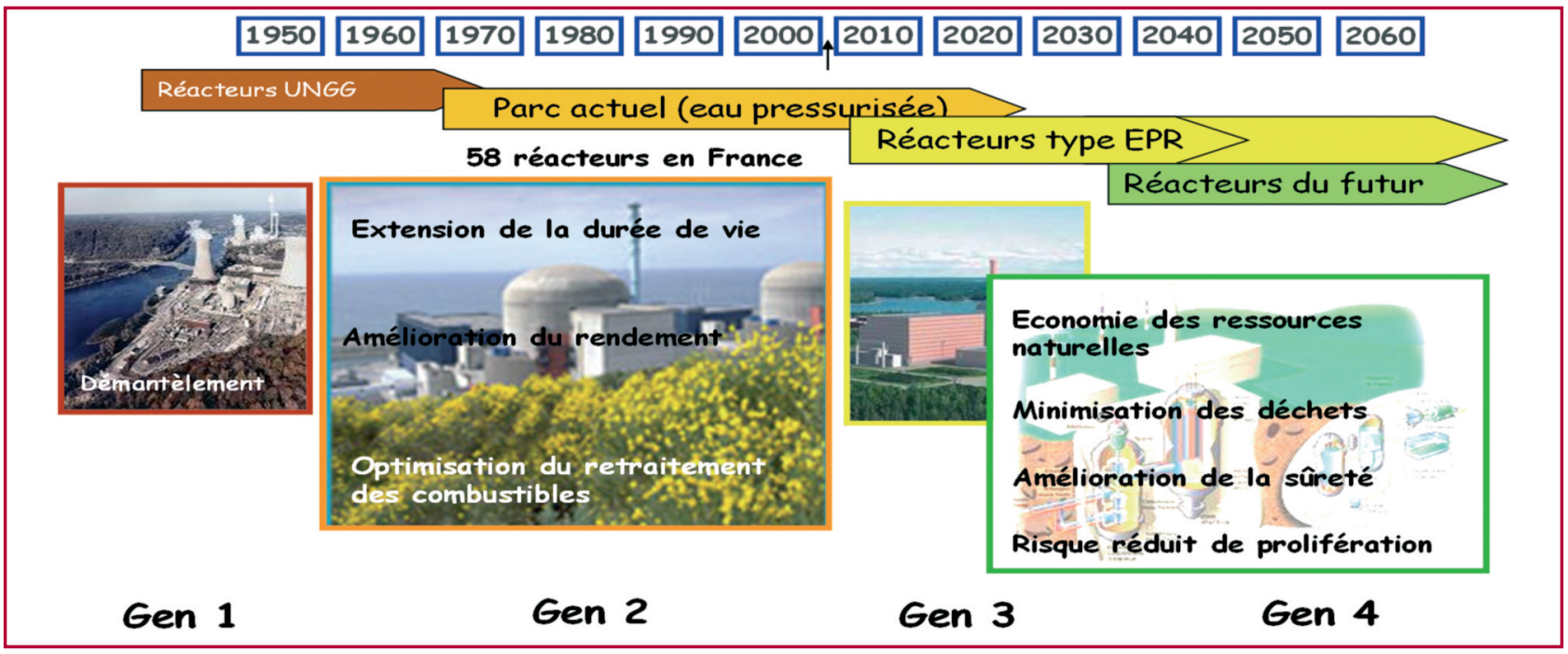

Fig. 1 - L'évolution des centrales nucléaires - Source : CEA 
- atteindre une bonne compétitivité compte tenu du service rendu ;

- présenter des garanties de résistance à la prolifération nucléaire.

\section{Les technologies étudiées au sein du forum international GEN IV}

Le cadre international de la coopération en matière de systèmes nucléaires de 4 ème génération est le GIF (Gen IV International Forum), dont l'objectif est la conduite des travaux de R\&D nécessaires à la mise au point de systèmes nucléaires (réacteurs et cycle du combustible) répondant aux critères de durabilité de l'énergie nucléaire.

\begin{tabular}{|ccc|}
\hline & France & \multicolumn{2}{c|}{ Royaume-Uni } \\
Chine & & Russie \\
Canada & Forum & UE \\
USA & international & Suisse \\
Brésil & Génération IV & Japon \\
Argentine & & Corée du Sud \\
& Afrique du Sud & \\
\hline
\end{tabular}

Fig. 2 - L'organisation du forum international GIF
Le GIF est une association intergouvernementale lancée en 2000 à l'initiative du "Department of Energy" des ÉtatsUnis. Il regroupe à ce jour treize membres ${ }^{1}$ engagés par la signature d'une charte dans laquelle ils reconnaissent l'importance du développement de systèmes futurs pour la production d'énergie nucléaire, ainsi que la nécessité à la fois de préserver au mieux l'environnement et de se prémunir contre les risques de prolifération (figure 2).

Le Forum a sélectionné six concepts, à neutrons rapides ou à neutrons thermiques, ${ }^{2}$ et a défini un programme de $R \& D$ afin d'apporter les innovations nécessaires pour leur déploiement.

On notera que dans la figure 3 récapitulant les six concepts, quatre réacteurs sont des réacteurs rapides cherchant à répondre aux objectifs de durabilité du nucléaire.

Deux réacteurs thermiques sont présents qui répondent à d'autres objectifs.

Le premier est le réacteur à eau supercritique, qui est une optimisation d'un réacteur bouillant où l'on essaye d'utiliser les propriétés de l'eau supercritique, pour atteindre des gains économiques potentiels et substantiels. Un article dédié explique l'état d'avancement de ce concept ainsi que les difficultés correspondantes. Aujourd'hui la faisabilité de ce concept n'est pas assurée. Le durcissement du spectre amené par la réduction du

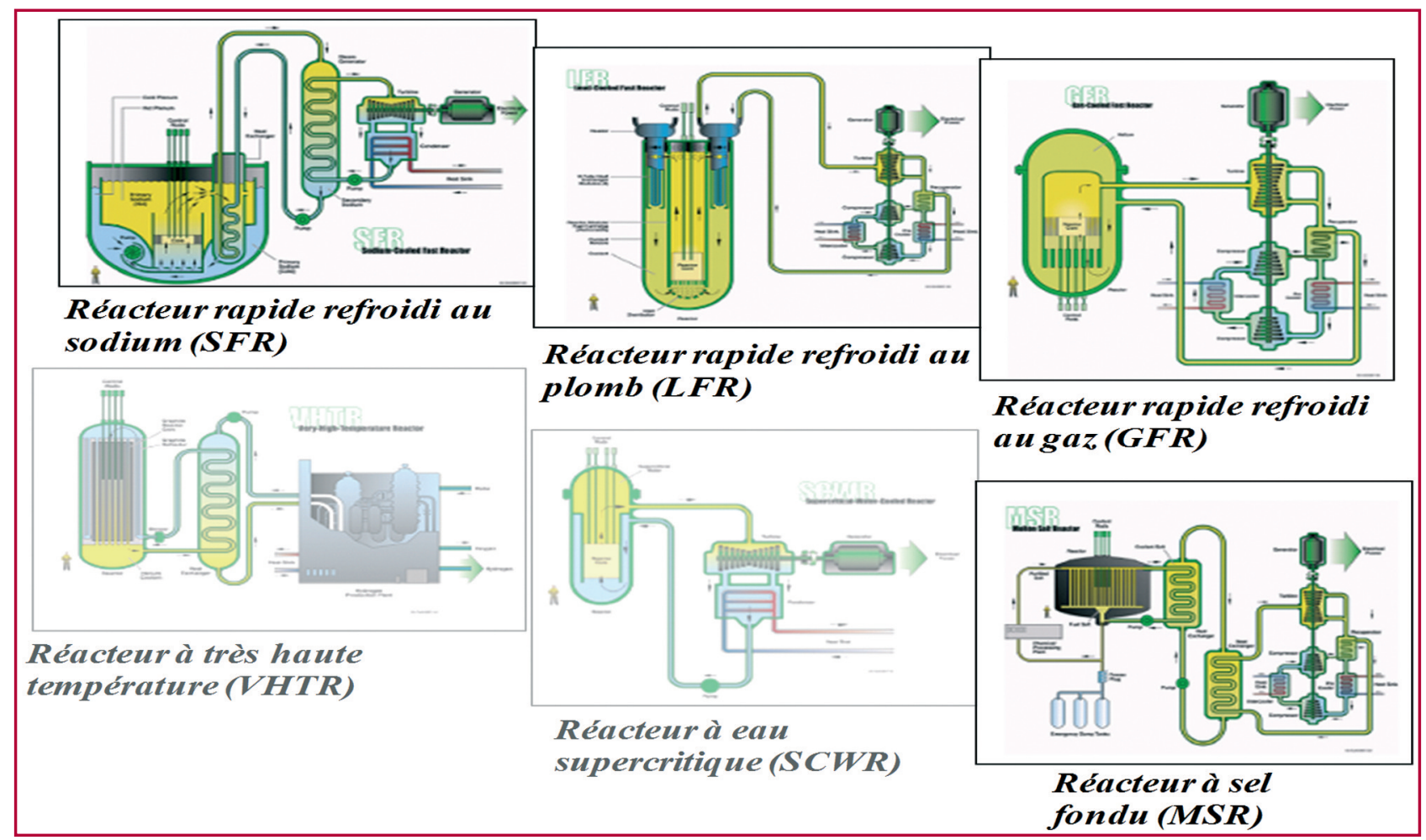

Fig. 3 - Récapitulatif des six concepts étudiés dans le cadre du forum GIF

\footnotetext{
${ }^{1}$ Afrique du Sud, Argentine, Brésil, Canada, Chine, États-Unis, Euratom, France, Japon, République de Corée du Sud, RoyaumeUni, Russie, Suisse.

${ }^{2}$ Neutrons rapides : Sodium-cooled fast Reactor (SFR), Gas-cooled fast Reactor (GFR), Molten Salt Reactor (MSR), Lead-cooled Fast Reactor (LFR). Neutrons thermiques : Super Critical Water-cooled reactor (SCWR), Very High Temperature reactor (VHTR).
} 
volume d'eau permet de le classer en réacteur épithermique, mais pas vraiment en réacteur rapide (voir l'article).

Le second est le VHTR, qui est le dérivé des réacteurs HTR développés dans les années 1980. Ces réacteurs fonctionnent avec un gaz comme caloporteur, ce qui permet d'atteindre des températures de sortie élevées. Le VHTR avait été introduit à l'origine du forum GIF dans les six concepts retenus, parce que les USA à l'époque plaçaient de grands espoirs dans un procédé lode/Soufre de production d'hydrogène. Celui-ci nécessite des températures de l'ordre de $1000^{\circ} \mathrm{C}$ que seul ce type de réacteur semblait alors pouvoir produire. Depuis le procédé a été totalement abandonné. De plus il a fallu revoir les températures à la baisse (aux environs de $750^{\circ} \mathrm{C}-850^{\circ} \mathrm{C}$ ), car les matériaux pour atteindre les températures initialement recherchées ne sont pas encore disponibles. En conclusion, on se retrouve avec un concept de HTR, qui a potentiellement des propriétés de sûreté intéressantes à condition de rester à de faibles puissances (inferieures à $300 \mathrm{MWe}$ ). Un article dédié explique l'historique de ces réacteurs et l'état d'avancement de leur technologie.

Pour les quatre réacteurs rapides restants, il faut noter que la maturité technologique de ces concepts est très variable. Or on peut considérer qu'il existe un lien fort entre la sûreté et la maturité technologique. Pour les RNR sodium, 19 ont déjà été construits et opérés dans le monde et plusieurs sont actuellement en projet ou en construction. Un article dédié explique l'état d'avancement de ces projets et des réacteurs en construction, dont deux devraient diverger très prochainement : un 500 MWe en Inde (PFBR) et un 800 MWe en Russie (BN 800), sans prétendre remplir tous les critères de sûreté de la quatrième génération.

Pour les autres rapides : plomb, gaz et sels fondus, l'état des connaissances est beaucoup plus faible. II n'y a jamais eu de réacteurs au plomb (mais une filière de réacteurs $\mathrm{Pb} / \mathrm{Bi}$ pour les sous-marins russes), ou de réacteur rapide en gaz, et il y a eu deux réacteurs prototypes de $2,5 \mathrm{MWt}$ et $8 \mathrm{MWt}$ à sels fondus.

Trois articles sont dédiés à chacune de ces technologies en expliquant l'historique, les avantages potentiels, les développements en cours, et les principales difficultés.

En conclusion, les états de développement des six concepts étudiés dans le GIF ne sont pas comparables.

Pour les cinq concepts autres que le RNR sodium, on reste au stade de la recherche de faisabilité. Des verrous technologiques restent à lever, en particulier souvent au niveau des matériaux. On trouvera ci-dessous, à titre d'exemple, les principaux verrous technologiques recensés dans les cinq articles dédiés à ces filières et pour lesquels des actions de R\&D sont engagées.

VHTR : - Conception de particules étanches au-delà de $1600^{\circ} \mathrm{C}$

- Matériaux (fonctionnement à haute température)

GFR : - Conception des éléments et de l'assemblage combustible

- Réalisation de la fonction d'évacuation de la puissance résiduelle
- Conception de l'enceinte rapprochée pour garantir une pression minimale en cas de brèche primaire

LFR : - Maîtrise des effets corrosifs et érosifs du plomb sur les structures

- Tenue au séisme

- Manutention et conditionnement des assemblages

MSR : - Maitrise des effets corrosifs du sel combustible

- Tenue des structures à haute température

- Conception des échangeurs intermédiaires

- Maitrise des moyens visant à extraire certains PF du sel combustible

- Réalisation et fiabilité de la fonction de vidange du sel combustible dans l'espace sous-critique

SCWR : - Matériau de gainage des éléments combustibles

- Conception du cœur (fort couplage neutronique thermo hydraulique, zones de mélange)

- Matériaux (fonctionnement à haute température, sous flux et dans un fluide très corrosif).

Pour le RNR sodium, et grâce à un REX important, la faisabilité du concept est démontrée. L'enjeu des études actuelles autour de ce concept est d'atteindre les ambitieux objectifs de sûreté assignés aux réacteurs de quatrième génération. C'est l'objet principal du projet ASTRID actuellement développé par le CEA (Voir article "Réacteur de 4ème génération ASTRID : I'organisation du projet et les principales orientations techniques" de M. Le Coz, RGN N³ 2013 mai-juin).

On peut simplement rappeler ici quelques progrès décisifs en termes de démonstration de sûreté sur ce projet ASTRID :

- cœur amélioré à coefficient de vidange négatif. Ce cœur constitue une avancée essentielle dans le domaine de la sûreté et confère au réacteur un comportement naturel favorable en cas de perte de refroidissement;

- système de conversion d'énergie à générateurs de vapeur modulaires (pour limiter les effets d'une éventuelle réaction sodium-eau), ou à échangeurs sodium-azote (pour éliminer totalement la présence d'eau à proximité du sodium) ;

- grande inertie thermique, convection naturelle, systèmes d'évacuation de la puissance résiduelle passifs et actifs, redondants et diversifiés (sources froides : eau et atmosphère) ;

- prise en compte des agressions externes dès la conception (séisme, inondation, chute d'avion...), avec suffisamment de marges pour garantir l'absence d'effet falaise :

- conception d'ensemble de l'architecture du réacteur garantissant une résistance accrue aux risques de prolifération.

Le démonstrateur ASTRID (figure 4) est l'étape-clé permettant de disposer de la démonstration industrielle d'un réacteur de 4ème génération, sachant que dans les décennies qui viennent, il faudra stabiliser les stocks de produits disponibles (multirecyclage du plutonium), économiser les ressources naturelles (en particulier si l'accès à l'uranium naturel devient difficile) et éventuellement transmuter les actinides mineurs. Un article dédié regarde du point de vue économique les possibilités d'introduction dans un parc de réacteurs à eau de ces réacteurs rapides 


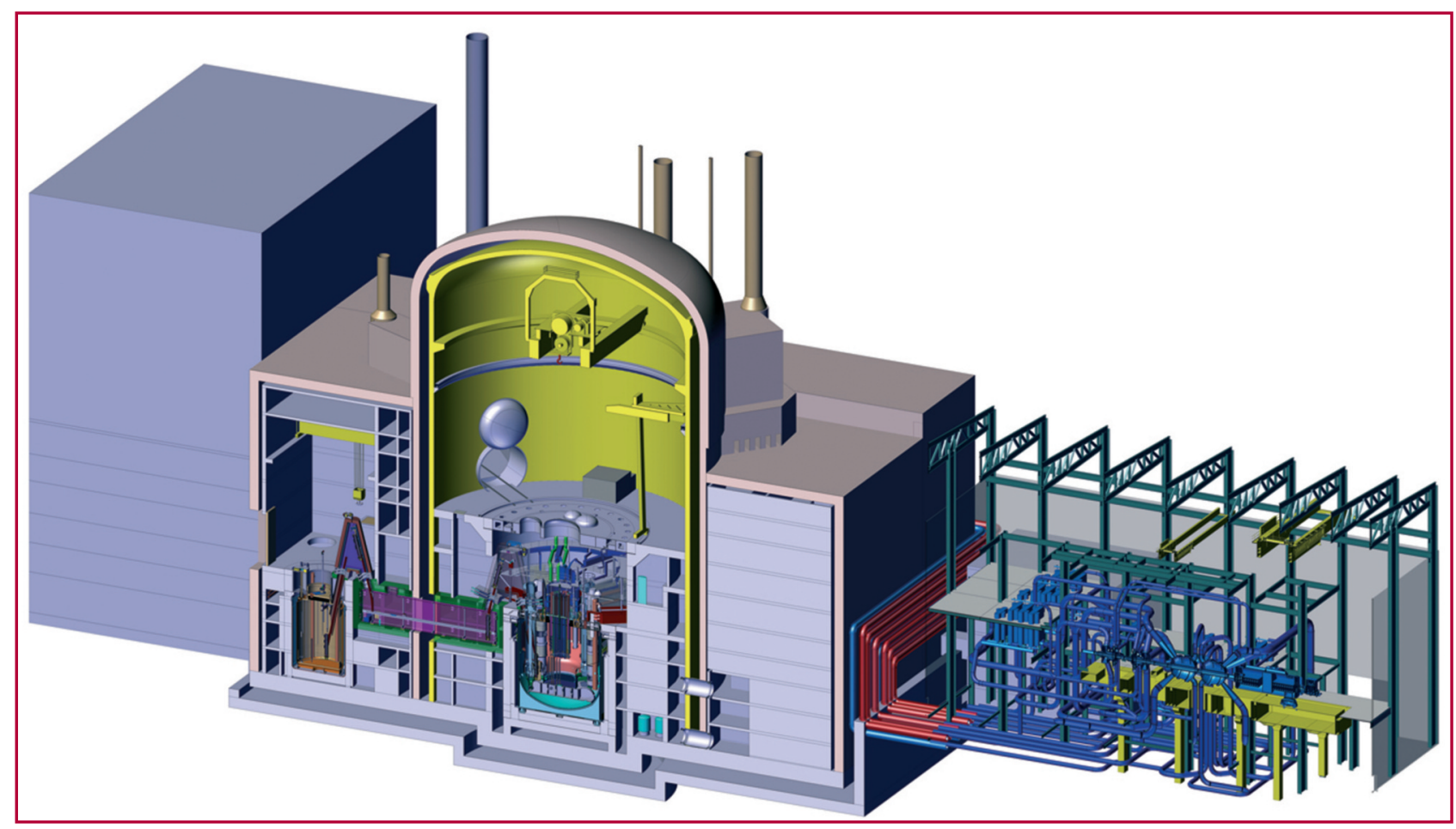

Fig. 4 - Vue en coupe du réacteur ASTRID

qui seront d'un coût d'investissement plus élevé au départ, mais qui apportent des gains sur la durabilité et des gains économiques sur le cycle (plus besoin de mines d'uranium et d'usines d'enrichissement pour ces réacteurs).

\section{- Les petits réacteurs modulaires (Small Modular Reactors)}

Historiquement les réacteurs de puissance ont toujours vu leur puissance augmenter, car cela conduisait à une baisse du coût du MWh produit. Les SMR ne se classent donc pas dans la logique des réacteurs de deuxième, troisième et quatrième génération. Ils se classent dans une logique de recherche de taille optimale par rapport aux demandes du marché et aux demandes des décideurs économiques.

Les puissances importantes actuellement atteintes, sont parfois trop fortes pour les réseaux, pour des pays de faible électrification ou pour des zones isolées. Pour ce marché, des réacteurs de plus faible puissance seraient nécessaires. De plus, ces petits réacteurs nécessitent un investissement initial moins important, pour un planning de réalisation plus court.

Plusieurs pistes sont donc explorées, pour proposer des réacteurs modulaires de petite puissance, en tentant de ne pas trop pénaliser le coût final du MWh proposé :

- profiter de la petite taille qui simplifie certains problèmes de sûreté, comme par exemple l'évacuation de la puissance résiduelle, et qui rend la conception et le fonctionnement plus simple.
- Profiter d'un effet de série en fabriquant ces réacteurs de manière modulaire, avec un maximum de constructions en usine.

- Avoir des gains importants sur les plannings de réalisation, et avoir un investissement de départ plus faible et donc plus accessible.

- Par une conception modulaire, pouvoir étaler l'investissement dans le temps, sur un même site.

Ces réacteurs de petite taille, existent déjà : ce sont les réacteurs embarqués de la propulsion navale : sous-marins et brise-glaces russes, sous-marins américains, porteavions et sous-marins français. II est donc logique que la réflexion s'organise prioritairement sur la base de ces petits réacteurs type PWR, pour lesquels un retour d'expérience important est déjà disponible.

Le DOE a relancé par un appel d'offre les recherches dans ce domaine. On trouvera dans ce RGN un article expliquant le programme américain de développement de ce concept SMR.

Les Français ont aussi lancé un programme de recherche. Un article explique les objectifs de ce programme ainsi que son état d'avancement. Et pour conclure, on trouvera un dernier article de DCNS sur la construction modulaire et les trente ans d'expérience dans ce domaine de la construction navale française.

II ne reste qu'à vous souhaiter une bonne lecture, de ces onze articles permettant de tenter d'avoir une vue générale de l'état des recherches et des développements pour ces réacteurs du futur. 